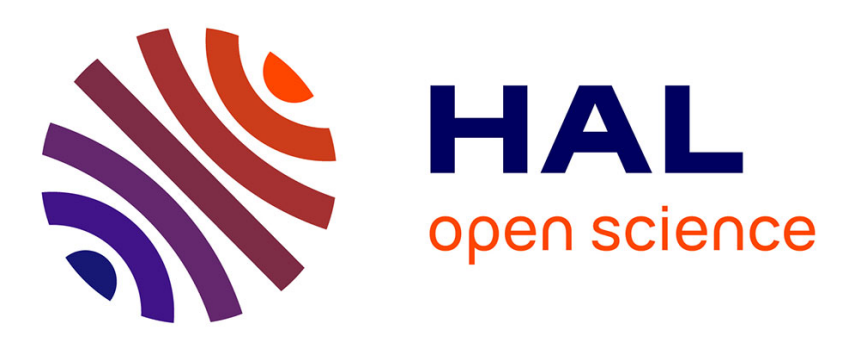

\title{
Trasformazioni urbanistiche in Sicilia alle origini della Provincia. Riflessioni sul ruolo di Roma Lorenzo Campagna
}

\section{To cite this version:}

Lorenzo Campagna. Trasformazioni urbanistiche in Sicilia alle origini della Provincia. Riflessioni sul ruolo di Roma. KTĖMA Civilisations de l'Orient, de la Grèce et de Rome antiques, 2019, La rhétorique de la diplomatie en Grèce ancienne, 44, pp.123-143. halshs-02444203

\section{HAL Id: halshs-02444203 \\ https://shs.hal.science/halshs-02444203}

Submitted on 17 Jan 2020

HAL is a multi-disciplinary open access archive for the deposit and dissemination of scientific research documents, whether they are published or not. The documents may come from teaching and research institutions in France or abroad, or from public or private research centers.
L'archive ouverte pluridisciplinaire HAL, est destinée au dépôt et à la diffusion de documents scientifiques de niveau recherche, publiés ou non, émanant des établissements d'enseignement et de recherche français ou étrangers, des laboratoires publics ou privés. 

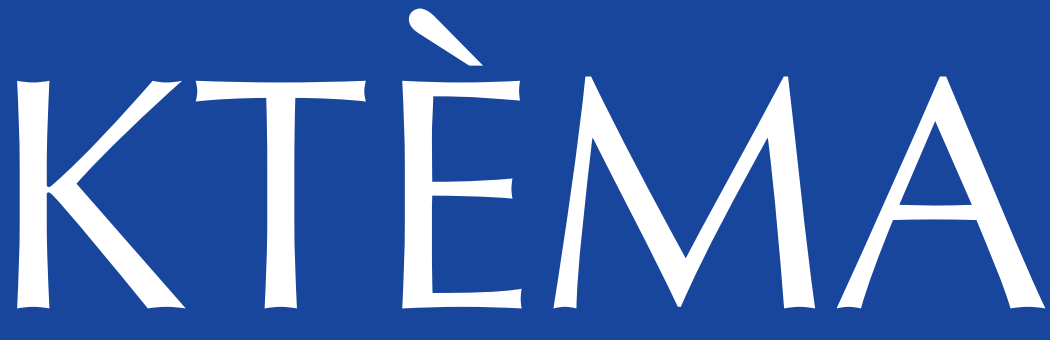

\section{CIVILISATIONS DE L'ORIENT, DE LA GRÈCE ET DE ROME ANTIQUES}

\section{La rhétorique de la diplomatie en Grèce ancienne}

Cinzia BeARzot, Laura Loddo

Laura LoDDo

Cinzia BeArzot

Paolo A. Tuci

Francesca Gazzano

Nicholas D. Cross

Davide Amendola

Jonathan R. W. PraG

Lorenzo CAMPAGNA

Cristina SORACI

Edith Foster

Walter LAPINI

Sylvain PERROT

Gianpaolo Urso

Anne JACQuemin
Political Exiles and Their Use of Diplomacy in Classical Greece

À propos du parallélisme entre deux discours d'ambassade à Sparte (Xén. Hell. V, 2, 12-19 et VI, 1, 4-16). 23

The Speeches of Theban Ambassadors in Greek Literature (404-362 B.C.)................ 33

Greek Ambassadors and the Rhetoric of Supplication. Some Notes ............................ 53

The (Im)balance of Power. Demosthenes' Complex Case

for an Alliance with the Megalopolitans.....

Presbeutikoi and Enteuktikoi Logoi in Hellenistic Interstate Relations.

Some Further Thoughts from an Epigraphical Perspective (c. 306-205 B.C.). 87

\section{Sicile hellénistique et romaine}

I.Sicily, Open Scholarship, and the Epigraphic Landscape of Hellenistic/Roman Sicily.

Trasformazioni urbanistiche in Sicilia alle origini della Provincia.

Riflessioni sul ruolo di Roma.....

Cultes et politique dans la Sicile du i ${ }^{\text {er }}$ siècle av. J.-C.

Le cas de la Vénus Érycine et de la Cérès d'Henna

\section{Varia}

Athens' Political Failures in the Central Chapters of Book 4 of Thucydides 163

Note interpretative e testuali alla col. XXII del Papiro di Derveni

La place de la musique dans la politique culturelle de Téos

dans la première moitié du $\mathrm{II}^{\mathrm{e}}$ siècle avant notre ère

Catilina legatus. Considerazioni su un discusso frammento sallustiano.

La dédicace aux Muses d'Eurydice fille de Sirrhas

(Plutarque, Sur l'éducation des enfants, 20) 


\section{Trasformazioni urbanistiche in Sicilia alle origini della Provincia Riflessioni sul ruolo di Roma}

Riassunto-. Gli impianti urbani regolari della Sicilia proto-ellenistica hanno attirato da tempo l'attenzione degli studiosi, ma le incertezze sulla cronologia che ancora sussistono in molti casi hanno determinato interpretazioni discordanti del loro contesto storico e culturale. In passato tali interpretazioni sono state spesso guidate prevalentemente da considerazioni formali e metrologiche. Una rinnovata attenzione ai dati stratigrafici disponibili ha di recente portato a stabilire, almeno per alcuni casi (Tindari, Solunto, Finziade), una datazione successiva alla metà del III secolo a.C., nelle fasi iniziali dell'organizzazione della Provincia Sicilia. In tutti e tre i casi, inoltre, la pianificazione a maglia regolare dello spazio urbano si sovrappone ad un abitato precedente, con evidenti modifiche nel regime delle proprietà. Occorre dunque avviare una riflessione sulle implicazioni sociali ed economiche connesse a queste trasformazioni, come pure sugli attori politici coinvolti, valutando il ruolo giocato in tali processi da Roma.

Parole chiave-. urbanistica, Provincia Sicilia, Tindari, Solunto, Finziade

Aвstract-. Orthogonal urban layouts in early Hellenistic Sicily have long attracted the attention of scholars, but the uncertainties on chronology still existing in many cases have led to divergent interpretations of their historical and cultural context. In the past these interpretations have often been guided mainly by formal and metrological considerations. A renewed attention to the available stratigraphic data has recently led to establish, at least in some cases (Tyndaris, Soluntum, Phintias), a dating after the middle of the 3rd century BC, in the initial phases of organization of the Provincia Sicilia. Moreover, in these three cases, the regular layout of the urban space overlies a previous settlement, with obvious changes in the property regime. It is therefore necessary to start thinking about the social and economic implications connected to these transformations, as well as on the political actors involved, evaluating the role played in these processes by Rome.

Keywords-. town-planning, Provincia Sicilia, Tyndaris, Soluntum, Phintias

I processi di trasformazione che interessarono gli insediamenti della Sicilia tra l'età di Timoleonte e la definitiva annessione dell'isola a Roma costituiscono un capitolo piuttosto importante sotto il profilo specificamente urbanistico, ma offrono anche un punto di osservazione

\footnotetext{
* Il testo è una versione rielaborata e ampliata della relazione da me presentata alla giornata di studio "La Sicile des Verrines, actualités de la recherche et des publications" (Parigi, INHA, 21 aprile 2018) organizzata da Sylvie Pittia, che desidero ringraziare per l'invito a partecipare. Sono inoltre grato alla Redazione di Ktèma per avere accolto il contributo e ai due referee anonimi per aver contribuito a migliorarlo con i loro suggerimenti, nonché a Carmine Ampolo, Oscar Belvedere, Salvatore De Vincenzo, Leonardo Fuduli, G. Francesco La Torre e Alessio Toscano Raffa per l'autorizzazione alla pubblicazione delle immagini.
} 
tutt'altro che secondario di questo complesso e tormentatissimo periodo della storia siciliana ed in particolare delle prime fasi della Provincia Sicilia.

Alla conoscenza di questi processi le ricerche degli ultimi quindici o vent'anni hanno offerto significativi contributi, soprattutto per quanto riguarda una più accurata scansione cronologica tra i fenomeni che è effettivamente possibile ascrivere al periodo in questione e quanto invece va attribuito a fasi più avanzate della vita della provincia repubblicana. Mi riferisco in particolare ai ben noti interventi di ristrutturazione in forme monumentali di aree pubbliche e di residenze private rilevabili in molti centri dell'isola, intorno ai quali, dopo anni di ampie e vivaci discussioni tra sostenitori di posizioni rialziste e ribassiste, sembra essersi costituito un consenso abbastanza largo su datazioni non anteriori al pieno II sec. a.C., quando l'assetto della provincia sotto il profilo socio-economico, oltre che politico e amministrativo, era divenuto più stabile ${ }^{1}$.

In diverse città provinciali si avviò allora una sorta di omologazione del paesaggio monumentale favorita dall'adozione di modelli architettonici e stilistici comuni -, dietro la quale sembra di poter riconoscere dinamiche socio-economiche abbastanza simili all'interno dei corpi civici²; più nette rimangono invece, le differenze nell'articolazione degli impianti urbani, che riflettono evidentemente vicende insediative pregresse diverse da un caso all'altro.

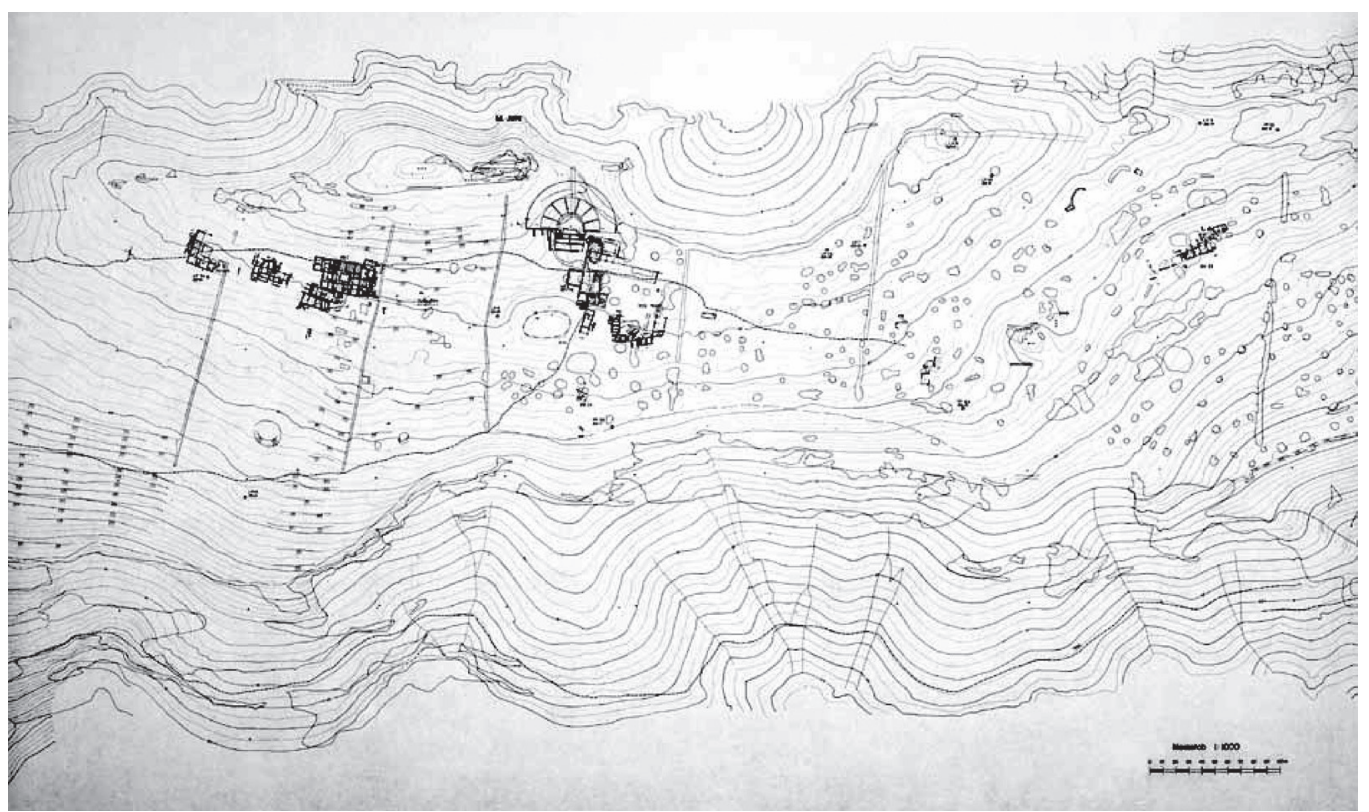

Fig. 1: Monte Iato. Pianta generale del sito in età ellenistica (H. P. Isler, 2006, «Monte Iato: scavi 2001-2003», in Guerra e pace in Sicilia e nel Mediterraneo antico (VIII-III sec. a.C.). Arte, prassi e teoria della pace e della guerra, Pisa, vol. II, fig. 280).

In alcuni centri, infatti, tali interventi si inseriscono nell'assetto preesistente, che viene allora dotato di una parure monumentale aggiornata, ma in maniera puntuale e senza stravolgimenti radicali dell'intero tessuto insediativo: il caso più emblematico è rappresentato da Monte Iato (fig. 1),

(1) Nell'ultimo ventennio la letteratura su questi temi è cresciuta in maniera esponenziale; mi limito pertanto a rinviare, anche per ulteriore bibliografia, ad alcuni contributi che affrontano ad un livello più generale le tematiche in discussione: Portale 2001-2002; Campagna 2003; La Torre 2004; Campagna 2006; Campagna 2007; Portale 2007; Prag 2009; Campagna 2011; De Vincenzo 2013b; Wilson 2013a; Wilson 2013b; Prag 2014; Portale 2015; Rocco 2015; Portale 2017. Per altri riferimenti si vedano i lavori citati nelle note successive.

(2) Su questi aspetti cfr. in particolare Prag 2003; Campagna 2007; Campagna 2011; Prag 2014. 
dove gli sviluppi più recenti della ricerca sul campo hanno permesso di delineare la sequenza di tappe del processo di trasformazione dell'abitato attuatosi tra IV e II sec. a.C. mantenendone sempre l'originaria impronta irregolare, forse risalente già ad epoca arcaica ${ }^{3}$. Al tipo di sviluppo delineato da Monte Iato si contrappone un insieme di casi, nei quali la monumentalizzazione tardoellenistica, in ambito pubblico e/o privato, appare invece iscritta in un tessuto caratterizzato da una suddivisione regolare e modulare, definita da un impianto viario ad assi ortogonali. Gli esempi meglio noti dal punto di vista archeologico sono offerti da Tindari, Solunto e Alesa, ma la stessa situazione, seppure con gradi di evidenza diversi, si rileva anche in altri centri, come Agrigento, Finziade, Panormos e Lilibeo. È evidente che la forma di questi centri, a differenza di Monte Iato, dipende da una pianificazione unitaria dello spazio urbano, in alcuni casi certamente più antica degli interventi edilizi tardo-ellenistici, come ad Agrigento ${ }^{4}$, in altri invece ad essi coeva, secondo quanto accertato, come vedremo, almeno a Finziade. Nella maggior parte dei casi, però, le relazioni cronologiche tra pianificazione e monumentalizzazione tardo-ellenistica sfuggono ancora ad una definizione condivisa per la mancanza di una documentazione archeologica adeguata, e infatti sulla data di origine di questi - e di altri - impianti urbani siciliani esiste ormai abbondante letteratura, con posizioni oscillanti tra gli inizi del IV e il pieno II sec. a.C. e, di conseguenza, con sostanziali divergenze di interpretazione riguardo alle matrici culturali e ai contesti storici di riferimento ${ }^{5}$.

\section{IMPIANTI “IPPODAMEI" IN SICILIA: PROBLEMI CRONOLOGICI E INTERPRETATIVI}

Ancora fino alle soglie di questo secolo, nell'ambito di una prevalente tendenza a riferire all'epoca della fondazione o comunque entro la fine del IV sec. a.C. la datazione di gran parte degli impianti regolari (Tindari, Solunto, Alesa, Lilibeo, Panormos), hanno prevalso i richiami alla tradizione ippodamea, declinati in varie sfumature verbali ed evocati, per così dire, "trasversalmente" sia per centri della Sicilia orientale (Tindari), che per insediamenti all'epoca ricadenti nel cuore dell'eparchia punica (Eraclea Minoa, Solunto, Lilibeo $)^{6}$.

Attorno a questi ultimi, d'altra parte, e sempre muovendosi entro orizzonti cronologici alti, è stata costruita la nozione di un'urbanistica regolare punica di Sicilia ${ }^{7}$, senza che tuttavia venissero esplicitate le ragioni che avrebbero condotto in questi casi ad adottare criteri di organizzazione dello spazio profondamente diversi da quelli rilevabili in centri coevi di altre regioni sottoposte a Cartagine, come Kerkouane o, nella stessa Sicilia, la Selinunte punica ${ }^{8}$.

(3) Si vedano, anche per la bibliografia precedente, Russenberger 2016; Russenberger 2017. Inoltre: Portale 2015, p. 718-725; PORTALE 2017, p. 164-166, 172-175.

(4) Sull'impianto urbano di Agrigento, oggetto di recentissime nuove acquisizioni, e sul suo rapporto con la monumentalizzazione delle aree pubbliche e abitative di epoca tardo-ellenistica, si vedano da ultimo BriEnZA, CALIÒ, FurCas et al. 2016; BrienzA, CALIÒ 2018.

(5) Per le questioni cronologiche relative agli impianti regolari delle città citate nel testo, nonché di altri centri siciliani di epoca tardo-classica ed ellenistica, rinvio alle ampie discussioni in LA TORRE 2009, LA TORRe 2013, part. p. 426-432, e De VinCenZo 2013b.

(6) Cfr. ad esempio Castagnoli 1963, p. 194-195; Castagnoli 1971, p. 136-137; Di Vita 1996, p. 299-307; Belvedere 1998, p. XXI; Di VITA 2002; più prudente ed articolata la posizione di Sommella 1987-1988. Più di recente, per Tindari: Belvedere, Termine 2005; per Solunto: Medeksza 1990; Mezzolani 1994, p. 154; Tusa 1999; per Lilibeo: Mistretta 2016, part. p. 125 e nota 18, ove si parla di un "piano ortogonale per strigas di matrice greca".

Sugli abusi dell'aggettivo "ippodameo", a fronte dell'esiguità di dati relativi all'attività di Ippodamo, ha di recente richiamato l'attenzione GRUET 2008, con importanti considerazioni e con riferimenti alla vasta bibliografia sul tema.

(7) Per Solunto e per Panormos cfr. Spatafora 2009; per Lilibeo Caruso 2013; Caruso 2014.

(8) Al riguardo cfr. De Vincenzo 2014, p. 240-243; De Vincenzo 2016a, p. 136; De Vincenzo 2016b, p. 211-213, ma già VASSALLO 2011, p. 61, nota 43. Sui caratteri dell'urbanistica regolare nel mondo punico si veda MezzoLANi 1994. 
Da più parti, infine, è stata invece sostenuta la possibilità che questi più recenti impianti regolari siciliani appartengano a fasi successive alla fine del primo o, talora, del secondo conflitto romanopunico' ${ }^{9}$. Tuttavia, lo slittamento della datazione a momenti successivi alla provincializzazione non ha comportato che Roma venisse chiamata in causa evocando un suo qualsivoglia coinvolgimento, e neppure che il mondo romano-italico venisse individuato come ambito culturale di riferimento per i casi siciliani; soltanto recentemente quest'ultima strada è stata additata da Salvatore De Vincenzo, che ha evidenziato la possibilità di riconoscere in alcuni impianti urbani (Solunto, Panormos, Lilibeo) l'adozione di unità di misura romane, arrivando così a sostenere non solo una datazione recenziore, ma anche una "matrice romana"

È chiaro che divergenze di tale portata potranno essere definitivamente sanate solo dall'apporto di nuovi dati archeologici utili a dirimere i problemi cronologici di base. Non è dunque mia intenzione, in questa sede, entrare nel merito delle interpretazioni cui ho appena accennato, se non per osservare come nella maggior parte dei casi la discussione si sia concentrata sugli aspetti formali e metrologici, certamente imprescindibili ma forse non sufficienti a far luce sul largo spettro di implicazioni, sia logistiche che politico-ideologiche, connesse alla scelta di organizzare - o di riorganizzare - una comunità adottando una pianificazione regolare a scala urbana.

\section{RIORGANIZZAZIONE DELL'IMPIANTO URBANO A TINDARI, SOLUNTO E FINZIADE:}

I DATI ARCHEOLOGICI

Vorrei allora provare ad indirizzare la riflessione in questa direzione, partendo dai tre casi di Tindari, Solunto e Finziade (figg. 2-4), che, allo stato attuale delle nostre conoscenze, a mio avviso si prestano meglio a sviluppare un ragionamento su questi aspetti. Per Finziade disponiamo - caso pressoché unico - di un'edizione sistematica dei recenti scavi sul Monte Sant'Angelo, che hanno permesso di delineare tempi e modalità del processo di trasformazione dell'abitato ${ }^{11}$. Per Tindari e Solunto le condizioni sono meno fortunate, ma possiamo avvalerci di alcuni lavori condotti in questi anni partendo da un riesame sistematico dell'evidenza archeologica, che hanno portato ad una ricostruzione a mio parere molto attendibile delle rispettive storie urbane ${ }^{12}$.

La possibilità di fare riferimento a questi contributi mi esime dal ripercorrere per esteso le dinamiche insediative dei tre centri, consentendomi di richiamare solo i dati utili alla discussione. Nel caso di Finziade (fig. 2), l'impianto ortogonale della città messo in luce dagli scavi e databile su basi stratigrafiche nei decenni successivi alla seconda guerra punica, è il risultato di una nuova pianificazione unitaria del sito, che ha obliterato un preesistente assetto insediativo riferibile con ogni probabilità all'epoca della fondazione del 282 a.C. ad opera del tiranno agrigentino Finzia ${ }^{13}$. Una situazione analoga si è andata delineando negli ultimi anni anche per Tindari (fig. 3) e Solunto (fig. 4), dove la maglia regolare si sovrappone a strutture più antiche e non può dunque risalire, come è stato a lungo sostenuto, all'epoca della fondazione dei due centri, rispettivamente agli inizi

(9) Cfr. soprattutto La Torre 2009; La Torre 2013; De Vincenzo 2013a; De Vincenzo 2013b; Portale 2015; De Vincenzo 2016b; Portale 2017; per Tindari cfr. già La Torre 2004.

(10) Cfr. De Vincenzo 2013b, p. 58 (Panormos), 101-103, 120 (Lilibeo), 112-113 (Solunto); De Vincenzo 2013a, p. 771-775; De Vincenzo 2016a; De Vincenzo 2016b. Per Lilibeo cfr. già Schmiedt 1963, p. 67-71.

(11) La Torre, Mollo 2013; per l'impianto urbano, in particolare, p. 80-112 (F. Mollo) e le considerazioni sviluppate, sempre all'interno dello stesso volume, in La Torre 2013. Per ulteriori dati sull'impianto urbano, emersi nel corso delle ricerche più recenti, cfr. Toscano Raffa, Venuti, Papale, Miano 2015.

(12) Tindari: La Torre 2004; Solunto: Portale 2006. Inoltre: Portale 2017, p. 168-170 (per Tindari) e 171-172 (per Solunto).

(13) La TORRe 2013. 


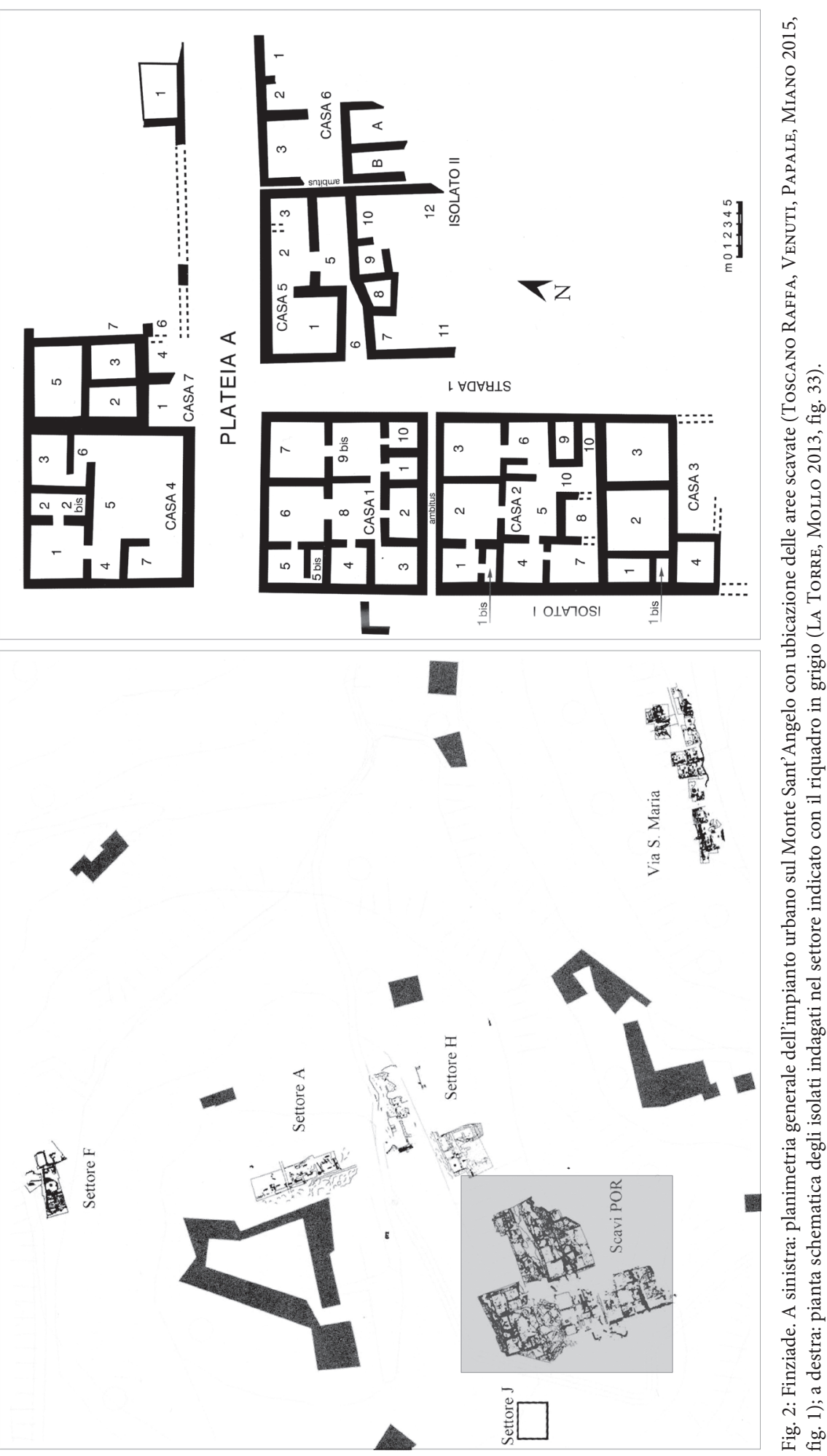




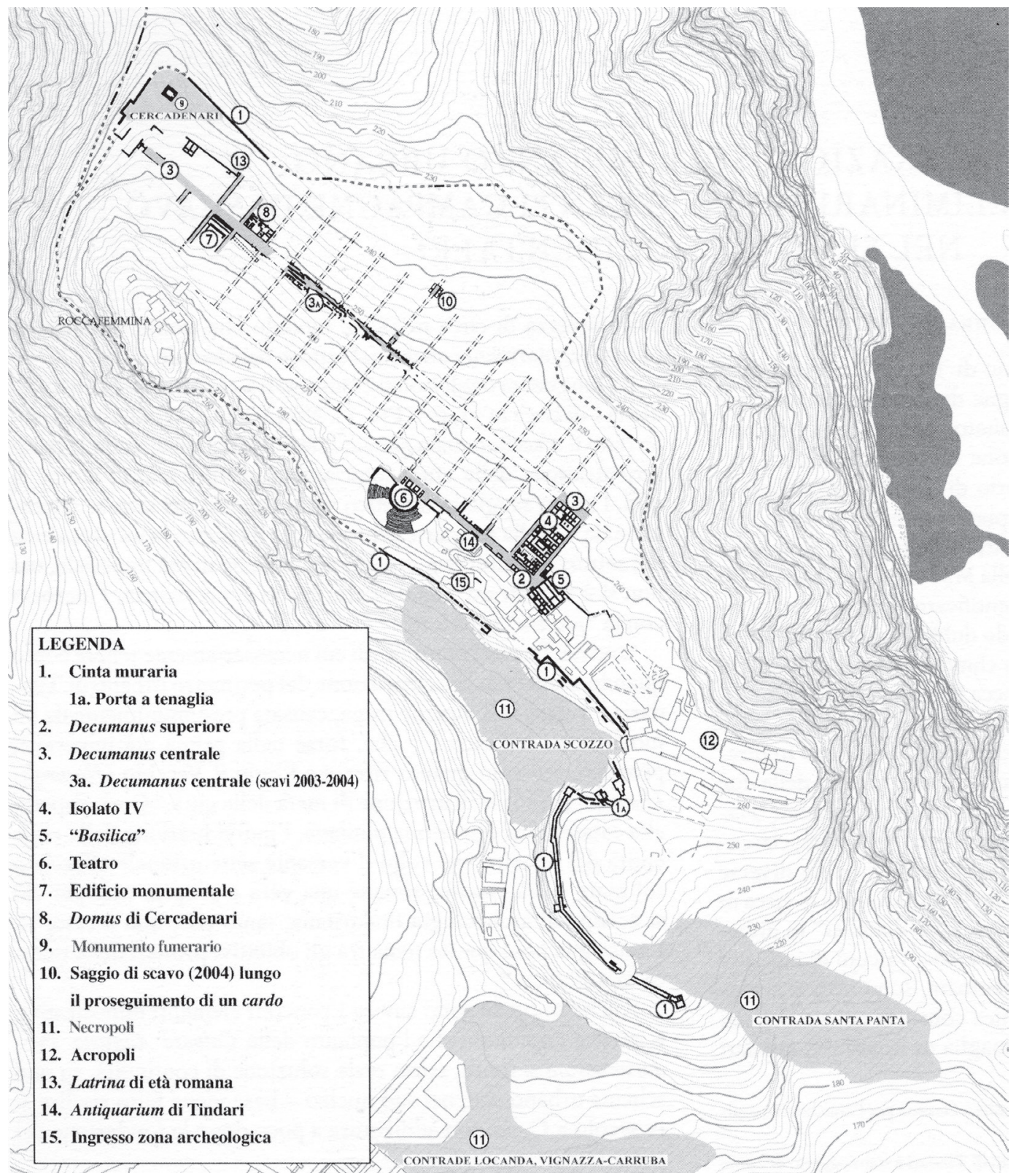

Fig. 3: Tindari. Planimetria generale (L. Fuduli, 2016, «Marmora Tyndaritana. Contributo allo studio della decorazione architettonica di Tindari in età imperiale», Römische Mitteilungen 122, fig. 1). 


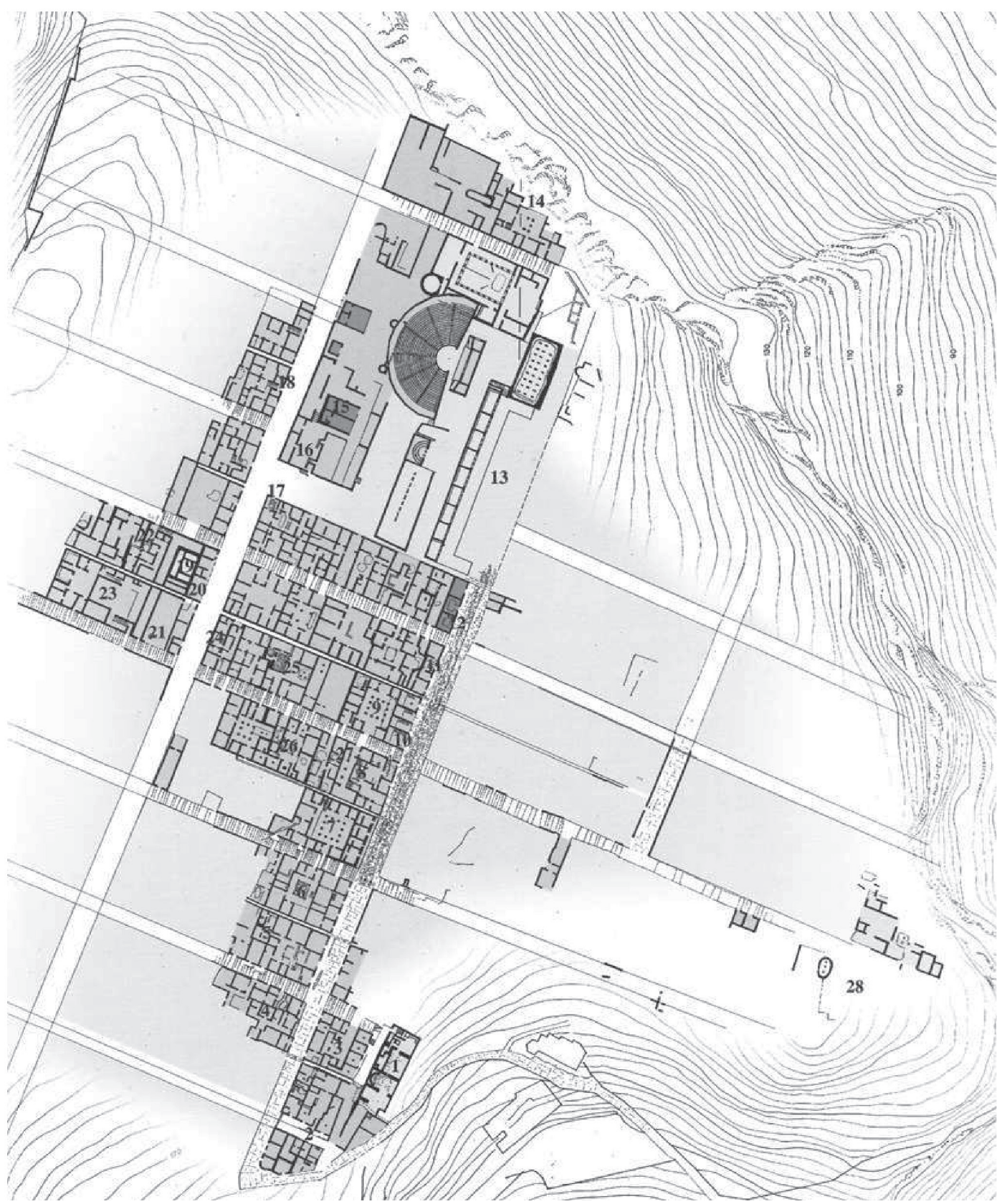

Fig. 4: Solunto. Planimetria generale (De Vincenzo 2016, Abb. 1). 
e nel tardo IV sec. a.C. ${ }^{14}$; a differenza di Finziade, però, i dati archeologici disponibili per stabilire la cronologia del nuovo piano sono più labili in entrambi i casi. Per Tindari, recentemente Chiara Portale ha richiamato l'attenzione sui resti di strutture più antiche in corrispondenza dell'insula IV dell'impianto regolare, in base alle quali per quest'ultimo sembrerebbe doversi assumere una data $a$ partire dal secondo quarto del III sec. a.C., la qual cosa, a mio parere, configura come scenario più plausibile il periodo successivo alla deditio della città ai Romani nel 254 a.C. ${ }^{15} \mathrm{Nel}$ caso di Solunto, le relazioni cronologiche tra le diverse preesistenze individuate in più punti del sito ${ }^{16} \mathrm{e}$ tra queste e l'impianto regolare sono più difficili da definire; la proposta più attendibile di ricostruzione delle fasi di trasformazione dell'abitato, a mio parere, è a tutt'oggi quella avanzata anche in questo caso da Chiara Portale, secondo la quale la redazione del piano regolare risalirebbe pure a Solunto agli anni successivi alla fine della prima guerra punica ${ }^{17}$.

Dal punto di vista formale e metrologico i tre impianti sono accomunati da caratteristiche simili già ampiamente esaminate in passato, sia per quanto riguarda l'articolazione degli assi viari principali e secondari e la disposizione per strigas, sia le proporzioni più contenute dell'isolato (rapporti compresi tra 1:2 e 1:2,5 ${ }^{18}$ : tratto, questo, che notoriamente ricorre in numerosi impianti, non solo della Sicilia, a partire da età proto-ellenistica, differenziandoli dalle proporzioni assai più allungate dell'urbanistica coloniale di età arcaica e classica ${ }^{19}$.

L'altro dato rilevante a fini del discorso riguarda la suddivisione degli isolati abitativi in lotti attuata in queste nuove pianificazioni, nitidamente individuata a Licata, sul Monte Sant'Angelo, dove i lotti non hanno subito modifiche successive ed hanno perciò mantenuto le dimensioni originarie (fig. 5$)^{20}$ : gli isolati indagati risultano infatti suddivisi in unità abitative con una configurazione planimetrica simile e la medesima estensione, circa $200 \mathrm{~m}^{2}$. La ripartizione interna dell'isolato prevista nella pianificazione è stata riconosciuta anche a Tindari nell'insula IV, l'unica

(14) Cfr. supra, bibliografia citata a nota 6. Una datazione alta dell'impianto regolare di Tindari, entro la fine del IV sec. a.C., è stata inoltre sostenuta in Spigo 2006 e Spigo 2008; per Solunto Spatafora 2009, p. 231-234, con riferimento ai risultati - a tutt'oggi inediti - di un saggio stratigrafico lungo una delle plateai (cd. via degli Ulivi), che ne attesterebbero l'esistenza già in epoche precedenti al III-II sec. a.C.

(15) Portale 2017, p. 169-170 e nota 108. Si tratta di resti di strutture murarie individuate nel secolo scorso durante lo scavo sistematico dell'insula, nel settore nord-ovest (in corrispondenza della più tarda casa B). L'anteriorità rispetto all'impianto dell'insula si deduce, oltre che dalle differenze di tecnica e di orientamento rispetto ai muri dell'insula, dal fatto che tali strutture dovevano proseguire verso ovest oltre il limite dell'isolato e al di sotto del cd. cardo E, al quale sono evidentemente precedenti. La datazione a fine IV-inizi del III sec. a.C. deriverebbe dai "reperti dallo strato di abbandonolivellamento per le costruzioni soprastanti” (ivi). Per notizie preliminari sulle fasi più antiche dell'Insula IV si veda CAVALIER 1970 , dove, alla fig. 1, è pubblicata una pianta schematica della parte dell'Insula IV comprendente le strutture più antiche.

(16) Sulle testimonianze di strutture più antiche individuate in diversi punti dell'area pubblica centrale rinvio, anche per la bibliografia specifica, a Portale 2017, p. 162-163, 171-172; cfr. anche De Vincenzo 2013b, p. 107, 110-111, 270-279, 282.

(17) Portale 2017, p. 171-172.

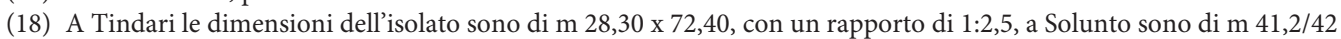
x 81,5/82, con un rapporto di 1:2: cfr. Belvedere, Termine 2005, rispettivamente p. 85-87 e 89. A Finziade è ricostruibile solo l'estensione del lato corto, $\mathrm{m}$ 28,30, mentre la misura del lato lungo doveva essere almeno di m 54,20: cfr. LA TORRE 2013, p. 422-423, con cauti ma suggestivi accostamenti alle proporzioni dell'impianto di Tindari.

(19) Proporzioni simili a quelle dei tre centri in esame, o ancora più compatte, presentano in Sicilia gli isolati dell'impianto di Cefalù (1:2: Belvedere, Termine 2005, p. 87, genericamente datato in età ellenistica, ma forse, secondo gli autori, più antico) e di Alesa (Tigano 2016, p. 139: rapporto di poco superiore a 1:1). In Italia meridionale si può citare il caso di Castiglione di Paludi (probabilmente 1:1,25: cfr. Brienza, Caliò, Lippolis 2011). Più sviluppate in lunghezza, anche se di poco, sono invece le proporzioni degli isolati di alcuni impianti comunemente datati al IV sec. o ad epoche più risalenti, come Morgantina (1:2,7 secondo La Torre 2013, p. 429; 1:3 secondo Belvedere, Termine 2005, p. 89) e Lilibeo (1:3,3 e 1:4: cfr. Mistretta 2016, p. 128), nonché Eraclea lucana (1:3: Belvedere, Termine 2005, p. 89), mentre nella rifondazione timoleontea di Camarina il rapporto è di 1:4 (Belvedere 1987, p. 7-8; Di Vita 2002). Per quanto riguarda invece le proporzioni più allungate, tipiche dell'urbanistica coloniale tardo-arcaica e classica, basti il riferimento a MERTENS 2006, p. 173-201, 340-354.

(20) Cfr. La Torre, Mollo 2013. 
TRASFORMAZIONI URBANISTICHE IN SICILIA ALLE ORIGINI DELLA PROVINCIA

131

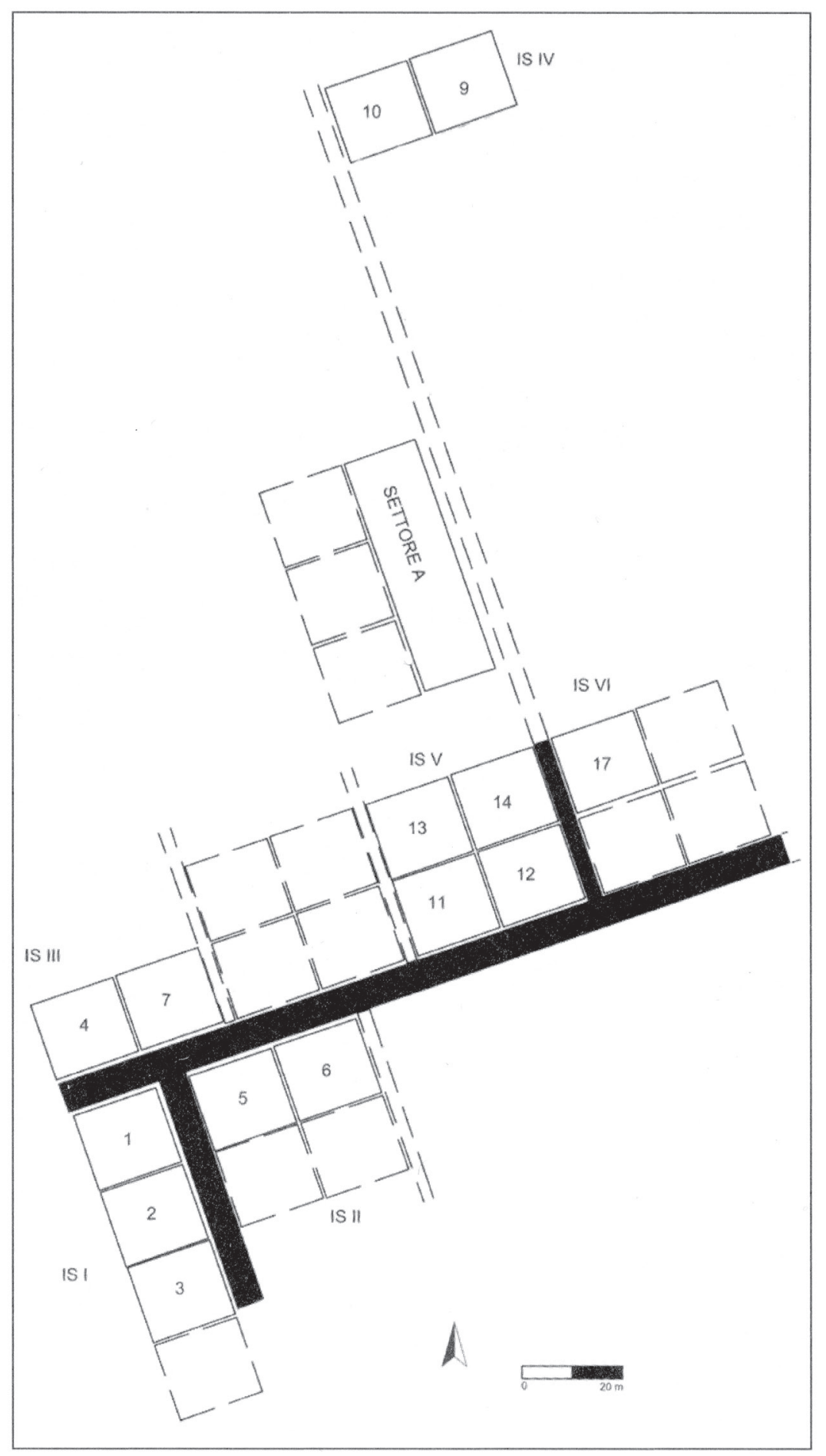

Fig. 5: Finziade. Ricostruzione della ripartizione dei lotti all'interno degli isolati (Toscano Raffa, Venuti, Papale, Miano 2015, fig. 7). 
esplorata sistematicamente: è stato qui possibile identificare un'articolazione in due file di quattro o cinque abitazioni delle stesse dimensioni, separate da un ambitus longitudinale (fig. 6) ${ }^{21}$. Questa prima suddivisione dell'isolato è stata poi trasformata, a partire dal II-I sec. a.C., da accorpamenti dei lotti originari nelle sontuose domus aristocratiche portate in luce dagli scavi ${ }^{22}$.

Non disponiamo invece di evidenze del genere a Solunto, perché negli isolati indagati non risulta siano state rinvenute tracce di ripartizioni precedenti rispetto all'assetto plano-volumetrico attualmente visibile, risalente a non prima del pieno II sec. a.C. ${ }^{23}$ L'esistenza di una situazione analoga a quella degli altri due centri non è tuttavia da escludere, in quanto potrebbe essere stata obliterata dalle imponenti ristrutturazioni successive.

\section{RIORGANIZZAZIONE DELL'IMPIANTO URBANO A TINDARI, SOLUNTO E FINZIADE: IMPLICAZIONI SOCIALI E POLITICHE}

Almeno per Finziade e per Tindari, dunque, la rimodulazione dell'impianto urbano coincide con una nuova suddivisione delle proprietà e con una ripartizione dei lotti abitativi che si manifesta con una connotazione egualitaria. In altri termini, la ridefinizione dell'insediamento che si materializza nel nuovo piano urbanistico riflette una forte discontinuità rispetto al passato non solo per quanto riguarda, evidentemente, il regime delle proprietà, ma anche più in generale l'assetto sociale e politico preesistente. Ed allora, rispetto a tale discontinuità la nuova pianificazione andrebbe a mio parere considerata come parte di un più ampio processo di ricostruzione della compagine civica. In definitiva, se ci limitiamo a descrivere tali fenomeni con termini quali crescita o espansione dell'abitato rischiamo di non cogliere a fondo la complessità di siffatte vicende, che sembrerebbero essere piuttosto identificabili come vere e proprie rifondazioni. Personalmente non escluderei che si sia trattato di fenomeni riconducibili alla nozione di sinecismo, non tanto nell'accezione (forse più nota) di costituzione di una polis tramite concentrazione in essa di più entità preesistenti, quanto piuttosto in quella, altrettanto attestata, di ripopolamento e rifondazione di una città, ricostituzione del corpo civico e dell'assetto politico-istituzionale ${ }^{24}$.

A questo punto, una volta che per operazioni di tale portata assumiamo, come è stato argomentato, una datazione dopo la metà del III sec. (Tindari; Solunto) o addirittura dopo la fine della seconda guerra punica (Finziade), diventa imprescindibile interrogarsi sulle circostanze, sulle ragioni e sugli attori di siffatti processi.

Da un punto di vista generale, credo che questi fenomeni non possano che essere letti in relazione al lunghissimo e pressoché ininterrotto periodo di conflitti che aveva devastato l'isola, conclusosi, solo temporaneamente, dopo la metà del III sec. a.C. con la creazione della vetus provincia. Al di là dei centri distrutti e spesso definitivamente abbandonati ${ }^{25}$, le fonti letterarie offrono diversi spiragli sulle traversie cui andarono incontro le comunità isolane negli anni di guerra, a causa di

(21) A tale ipotesi sull'originaria suddivisione interna dell'insula IV sono pervenuti indipendentemente LA Torre 2004 e Belvedere, Termine 2005, dove, a p. 87-89, si propone una ripartizione in due serie di cinque lotti ognuno di $\mathrm{m} \mathrm{14,50 \textrm {x }}$ 14,15 , o di quattro lotti di $\mathrm{m} 17,45 \times 14,15$, accordando tuttavia una preferenza alla prima ipotesi.

(22) Cfr. Bernabò Brea, Cavalier 1965.

(23) Cfr. Portale 2006. Una proposta di identificazione di una suddivisione originaria in lotti di m. 20 x 20 è stata formulata su un isolato campione da ItALia, Lima 1987, ma è priva di riscontri cronologici utili ai fini del nostro discorso; cfr. in proposito anche le osservazioni in Portale 2006, p. 55.

(24) Sulle diverse valenze del termine sinecismo cfr. MogGi 1975; Lombardo 1982, p. 853; Gallo 1982, p. 923 (con ulteriori riferimenti a nota 30); AMPolo 2001, p. 29 (L. Porciani).

(25) Si vedano ad esempio i casi di Selinunte, Monte Adranone, Montagna dei Cavalli/Ippana, Makella. Ancora utile al riguardo il quadro prospettato in BEJOR 1983, part. p. 350-352, e in WILsON 1985, p. 314-319. 

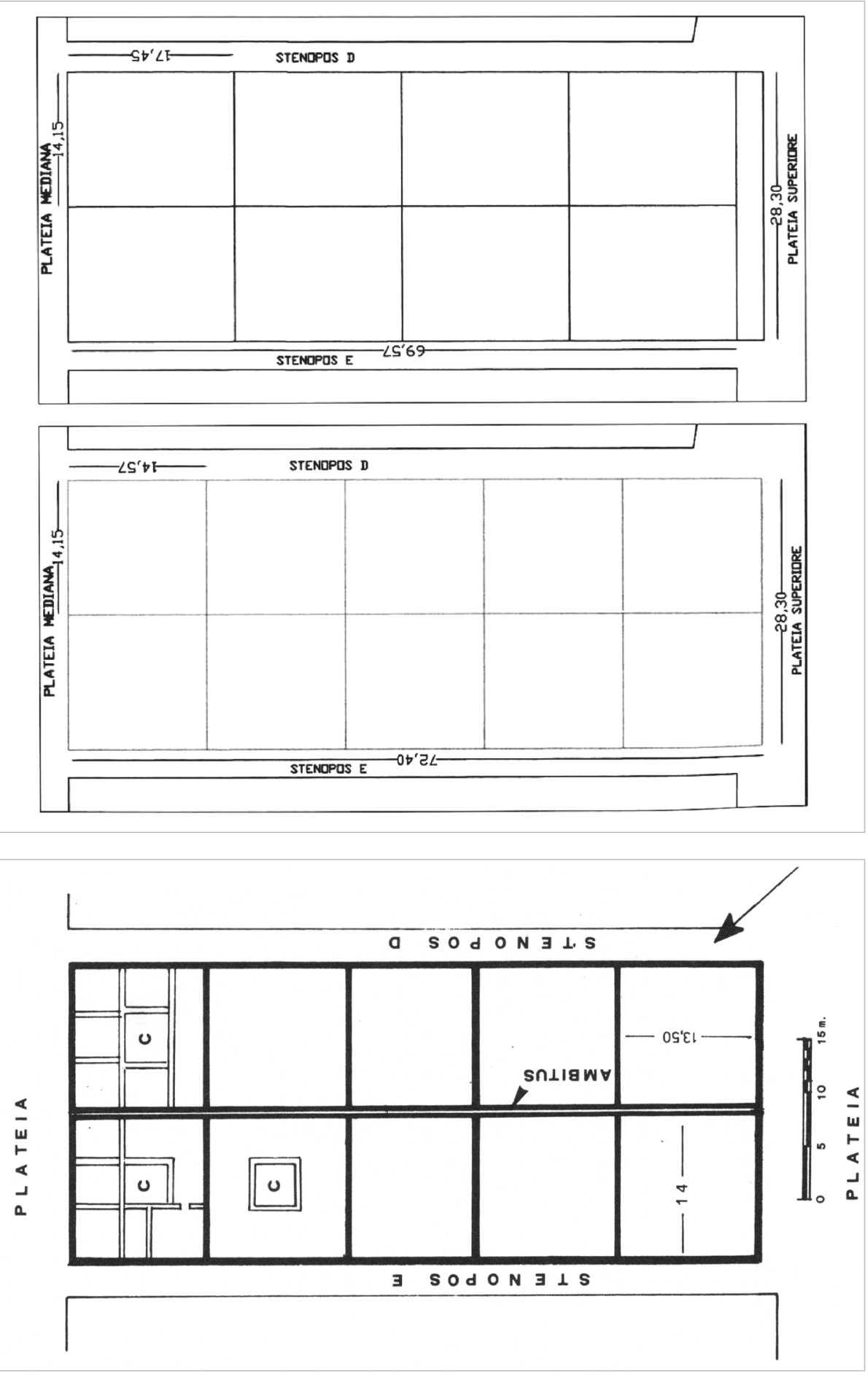

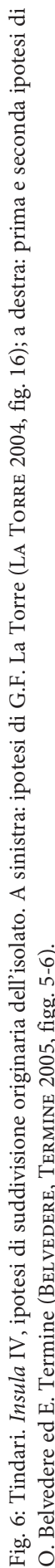


decimazioni di popolazioni, abbandoni e spostamenti forzati, o, viceversa, episodi di inurbamento o ancora altri fenomeni, come guerre intestine tra fazioni opposte, tutti con gravi ripercussioni sull'ordine interno ${ }^{26}$. Conosciamo bene, grazie allo straordinario dossier epigrafico entellino ${ }^{27}$, le vicende che tormentarono quella città ed è verosimile che peripezie analoghe abbiano funestato la vita di altre comunità. Nei casi qui in discussione, dalle fonti non si possono ricavare indizi utili a formulare ipotesi specifiche; tuttavia, gli interventi urbanistici ricostruibili dalla documentazione archeologica indurrebbero a pensare che non si trattò della restaurazione di uno status quo ante, quanto piuttosto di eventi che hanno mutato la composizione dei corpi civici sia da un punto di vista numerico che socio-economico.

D'altra parte, occorre chiedersi se tali operazioni siano la conseguenza solo di eventi traumatici congiunturali, cioè più direttamente legati allo scenario di guerra, o non anche di fenomeni di squilibrio e conflittualità sociali ed economiche in seno alla polis con radici più antiche, che le guerre potrebbero avere esacerbato ${ }^{28}$. Qualche spunto a tal proposito è possibile ricavare dal raffronto con quanto si rileva a Monte Iato, dove, come si è già detto, le trasformazioni monumentali incidono solo in misura circoscritta sull'organizzazione generale dello spazio urbano; sull'assenza a Iaitas di una rimodulazione urbanistica è tornato recentemente in maniera assai efficace Francesco La Torre $^{29}$, che ha proposto di collegarla alla persistenza del sistema sociale e politico locale dominato dal potere delle aristocrazie cittadine, che avrebbero ostacolato quella ristrutturazione di carattere "isonomico" che invece cogliamo almeno a Tindari e Finziade. Non è forse troppo peregrino, allora, immaginare che nei centri in esame si fosse verificato, per qualsivoglia ragione, un indebolimento dei gruppi di potere locali, non più in grado di mantenere l'ordine sociale interno e di opporre resistenze efficaci a rivendicazioni da parte di classi subalterne.

Quali che fossero le cause a monte, un altro aspetto rilevante della questione - finora, in verità, appena lambito dalla discussione, per quanto a me sembri ineludibile - riguarda il grado di coinvolgimento di Roma in queste vicende. In linea generale, è a mio parere assai improbabile che i processi che portarono alla rimodulazione degli impianti urbani si siano svolti in maniera autonoma, senza alcun intervento da parte del nuovo potere. Si tratta innanzitutto di operazioni complesse, che richiedono un'autorità politica forte in grado di governare l'intero processo, anche regolamentandolo sul piano legislativo ${ }^{30}$; non è un caso che operazioni di fondazione/rifondazione di città o di sinecismo risultino per lo più organizzate da potenze egemoni ${ }^{31}$. D'altra parte, esse necessitano di un notevole impegno organizzativo e di una consistente disponibilità di risorse. Ciò, a maggior ragione, quando si pensi al fatto che in tutti e tre i centri in esame il nuovo impianto urbano regolare viene attuato in condizioni orografiche tutt'altro che favorevoli, caratterizzate da suoli in forte pendio, con tutto quello che ne consegue in termini di opere preventive di sbancamento, terrazzamento, irreggimentazione delle acque: operazioni, tutte, che devono aver richiesto un consistente impegno finanziario ${ }^{32}$.

(26) Si vedano ad esempio Goldsberry 1973, p. 219-241; Gallo 1982, part. p. 931; Marasco 2007; Zambon 2008, p. 248-249.

(27) La letteratura sul corpus dei decreti di Entella, è ormai molto vasta e non può essere riportata qui in maniera esaustiva; mi limito pertanto a rinviare, oltre che ad AMPOLO 2001, al recente contributo di LOMBARDo 2018, che contiene tra l'altro ampi riferimenti alla bibliografia e alle acquisizioni più recenti sulla vicenda entellina.

(28) Sulla crisi sociale delle comunità civiche della Sicilia nel IV sec. a.C. cfr. da ultimo Corsaro 2016.

(29) La Torre 2013, p. 451; cfr. anche Portale 2017, p. 174.

(30) Cfr. ad esempio Gallo 1982, p. 930-931; Corsaro 2003, p. 380-381.

(31) Si veda al riguardo l'ampia esemplificazione presentata in Corsaro 2003, p. 380-384.

(32) Al riguardo cfr. LA TorRe 2009, p. 193. 
È difficile pensare che di tali risorse potessero disporre comunità che piuttosto immagineremmo con un'economia messa in ginocchio da decenni di guerra, né personalmente credo che, come già detto, si possano ascrivere interventi di questo genere a programmi delle aristocrazie locali ${ }^{33}$.

Purtroppo, anche per questo aspetto, non disponiamo di alcuna informazione specifica per i centri in esame; può essere, però, di qualche utilità richiamare le poche ma significative attestazioni desumibili dalle fonti scritte relativamente al ruolo giocato da Roma in vicende di ricostruzione/ ripopolamento di singoli centri siciliani durante o subito dopo la prima e la seconda guerra punica.

\section{INTERVENTI DI MAGISTRATI ROMANI PER RICOSTITUIRE I CORPI CIVICI}

La prima attestazione riguarda l'ormai notissimo caso del sinecismo di Entella e dell'epimeletas Tiberio Claudio Anziate durante o a ridosso della prima guerra punica ${ }^{34}$. Premetto che le peripezie subite dagli Entellini, così come si ricostruiscono dai decreti, configurano un caso diverso rispetto a quelli qui in discussione; se faccio riferimento ad esse, è per le implicazioni relative alla presenza ad Entella, durante il processo di rimpatrio e di riorganizzazione della comunità, di un rappresentante del governo di Roma. È verosimile che il personaggio, chiaramente identificato dall'onomastica come Romano, abbia avuto, come ritengono i più, un ruolo di carattere prevalentemente logistico e organizzativo a livello locale nella gestione del processo sinecistico, ma certamente non agiva per un'iniziativa individuale ma in virtù di un incarico conferitogli da Roma, forse $a d h^{35} c^{35}$; del resto, un altro e forse più indicativo segno dell'intervento di Roma nella vicenda entellina, a livello di assetto istituzionale, è stato più volte riconosciuto nella presenza della coppia arcontale a capo della città durante le fasi più delicate del processo ${ }^{36}$.

Il secondo caso, altrettanto noto, riguarda la città di Agrigento a conclusione della seconda guerra punica e l'operazione ivi condotta dal pretore T. (?) Manlius (Cic. Verr. 2.123-124) ${ }^{37}$ : questi, dopo che gli abitanti della città erano stati in parte trucidati, in parte venduti schiavi da Levino ${ }^{38}$, la ripopolò, per decisione del Senato romano, con il trasferimento forzoso di coloni dedotti, secondo l'interpretazione corrente, de oppidis Siculorum. Come si sa, sono tuttora aperti i problemi relativi all'identificazione del pretore e alla data dell'episodio ${ }^{39}$, ma ai fini del nostro discorso ciò che importa sottolineare è che anche in questo caso Roma intervenne direttamente, seppure con misure diverse, a ristabilire le compagini cittadine dopo i gravissimi dissesti causati dalle vicende belliche. Le nuove condizioni di forzata coabitazione tra coloni e veteres Agrigentini dovettero essere

(33) Importanti considerazioni sull'impoverimento delle comunità cittadine dopo la prima guerra punica in PoRTALE 2015, p. 724-725.

(34) SEG 30.1120. Nella nuova numerazione del dossier entellino il decreto in onore di Tiberio Claudio Anziate è il B1: cfr. Ampolo 2001, p. 19. Per la bibliografia generale sui decreti cfr. supra, nota 27.

(35) Cfr. soprattutto Corsaro 1982 e Corsaro 2003; inoltre Gallo 1982, p. 924-925; Mattioli 1995; Lombardo 2018, p. 488, 495.

(36) Cfr. Corsaro 2003, p. 384-393, con discussione della questione e ulteriore bibliografia. Di diverso avviso AnAST ASI 1999, p. 404-405, е ZамвоN 2008, p. 249, 258, che attribuiscono al sinecismo entellino un carattere autonomo e spontaneo.

(37) Cum Agrigentinorum duo genera sint, unum veterum, alterum colonorum quos T. Manlius praetor ex senatus consulto de oppidis Siculorum deduxit Agrigentum.

(38) Liv. 26.40.13.

(39) Si tratterebbe, secondo i più, del pretore nel 197 a.C., L. Manlius Vulso: cfr. ad esempio GabBa 1959, p. 310, nota 10; Manganaro 1964, p. 425; Goldsberry 1973, p. 240; Manganaro 1980, p. 422-423, 456, nota 44; Lombardo 1982, p. 858, con altri riferimenti a nota 40; Corsaro 2003, p. 394. In alternativa, ipotesi di identificazione con un L. Manlius inviato dal Senato ad Olimpia nel 208 a.C. (Liv. 27.35.3-4: vedi infra): Rizzo 1968-1969, p. 375-376, nota 27; Gallo 1982, p. 932 (dubitativamente). Infine Prag 2007b, p. 292 e 296: possibile correzione del nome nel testo ciceroniano ed identificazione con C. Mamilius, pretore del 207. 
regolamentate, come dimostrano le leggi per la cooptatio del senato locale stabilite pochi anni dopo, ancora una volta dall'alto (vicenda, questa, replicata peraltro ad Eraclea da P. Rupilius dopo la prima guerra servile $)^{40}$. È dunque Roma a governare l'intero processo, entro il quale è verosimile includere anche la risoluzione di altri problemi generati dall'insediamento dei nuovi abitanti, primo tra tutti la ridefinizione delle proprietà private.

Che le misure adottate nei confronti di Agrigento rispondessero a più generali preoccupazioni di Roma riguardo alle condizioni demografiche delle comunità siciliane dopo la seconda guerra punica, sembrerebbe confermato dall'appello che il Senato, in occasione dei giochi olimpici del 208 a.C., rivolse per il tramite di L. Manlius agli esuli siciliani perché tornassero in patria, con la promessa che sua omnia iis, quae ante bellum habuissent, reddere populum Romanum ${ }^{41}$. Probabilmente questo Manlius e il responsabile della deduzione di nuovi coloni ad Agrigento non sono la stessa persona, come invece riteneva F.P. Rizzo ${ }^{42}$, il quale peraltro intendeva la costruzione del passo ciceroniano sulla vicenda agrigentina diversamente dalla communis opinio, riferendo de oppidis Siculorum non alla provenienza dei coloni trasferiti in quella città, bensì al contenuto specifico del senatoconsulto (ex senatus consulto de oppidis Siculorum $)^{43}$. Una lettura, questa, che iscriverebbe l'operazione di ripopolamento di Agrigento in un provvedimento del Senato di diversa portata, riguardante un più vasto numero di oppida che versavano in condizioni critiche; indipendentemente dai problemi prosopografici e cronologici cui si è accennato, tale lettura a me non pare del tutto infondata, benché presupponga una linea di intervento da parte di Roma ad una scala ben più estesa, non altrimenti attestata dalle fonti scritte.

Gli episodi cui ho fatto riferimento, seppure isolati nella documentazione letteraria ed epigrafica, mi sembra riflettano una condotta analoga da parte di Roma nei confronti delle comunità siciliane già durante o dopo la prima guerra punica e poi, con maggiore evidenza, dopo la seconda: una condotta non certo ispirata da una politica di non ingerenza o di laissez faire ${ }^{44}$, bensì determinata non solo a sorvegliare - cosa che mi parrebbe abbastanza scontata - ma anche a guidare direttamente, se non addirittura a sollecitare, i processi di ricostituzione dei corpi civici locali.

Il contesto storico entro il quale vanno considerate queste scelte di Roma è quello, per così dire di sperimentazione e in divenire, delle fasi iniziali dopo la conquista, ben diverso dall'assetto della provincia di II-I sec. a.C., che conosciamo meglio; come si sa, il periodo che precede l'organizzazione imposta alla Sicilia da Levino nel 210-207 a.C. presenta ancora molti punti oscuri ${ }^{45}$. In queste fasi, a fronte di una conquista che poneva Roma in condizioni nuove rispetto alle esperienze pregresse di gestione dei territori nella penisola, occorre chiedersi quali fossero le valutazioni che guidavano le scelte relative ai centri urbani dell'isola.

Quali che fossero le intenzioni di Roma riguardo alla Sicilia dopo la pace del 241 a.C., non c'è dubbio che una preoccupazione preminente dovesse essere quella della difesa del territorio, soprattutto in vista di una recrudescenza del pericolo punico sia sul fronte siciliano che su altri fronti ${ }^{46}$; in tal senso, l'importanza del controllo delle coste e soprattutto dei porti difficilmente sarà stata sottovalutata. In questo quadro va probabilmente intesa la decisione del 227 a.C. di inviare nell'isola, per la prima volta, un praetor, forse preceduta già a partire dal 241 a.C. - se è vera la

(40) Gaвва 1959.

(41) Liv. 27.35.3-4; cfr. Marasco 2007, p. 182-183.

(42) Supra, nota 39.

(43) Rizzo 1976-1977, p. 206. La stessa lettura si trova già in Merguet 1877-1884, vol. 3, p. 491, s.v. "oppidum".

(44) È questa invece l'opinione espressa da ZAMBon 2008, p. 249, e più volte da A. Pinzone: cfr. PInzone 1999, p. 471; Pinzone 2000, p. 857; Pinzone 2004, p. 32-34.

(45) Sulle prime fasi della provincia: Crawford 1990, part. p. 92-96; Mattioli 1995; Pinzone 1995; Serrati 2000; Prag 2011; Prag 2012.

(46) Serrati 2000, p. 126-127. 
notizia riferita da Appiano (Sik. 2,6) - dall'invio annuale di uno strategos ${ }^{47}$; ma è significativa, in proposito, l'ipotesi più volte proposta riguardo alla costituzione della lega delle diciassette città insignite del privilegio di chrysophorein al santuario di Venere Ericina e incaricate, al tempo stesso, di fornire uomini per la guarnigione del tempio ${ }^{48}$ : se, com'è stato sostenuto con buoni argomenti, l'istituzione della lega risale ai primi anni dopo la pace del 241 a.C., essa potrebbe riflettere un precoce tentativo di controllare la Sicilia occidentale attraverso una lega di città fedeli ${ }^{49}$. Pertanto, che in tali circostanze i centri urbani, soprattutto, ma non solo, quelli costieri, assumessero agli occhi della potenza egemone un ruolo strategico è a mio parere abbastanza verosimile: si potrebbe allora comprendere un interesse da parte di Roma a rivitalizzare centri in condizioni di crisi demografica o comunque prostrati dalle guerre; ma anche ad intervenire in città minate al loro interno da situazioni sociali e politiche instabili o conflittuali, che avrebbero potuto degenerare e rappresentare un potenziale pericolo.

Al di là delle questioni di difesa, sicurezza e mantenimento dell'ordine, è stato opportunamente osservato che il ripopolamento e la stabilità socio-economica dei centri urbani dovevano apparire a Roma come una necessità anche in relazione all'organizzazione della fiscalità dell'isola, forse non solo dopo la seconda guerra punica ma anche da prima $^{50}$; sicché a qualcuno non è sembrato improprio attribuire a Roma, dopo la seconda guerra punica, un vero e proprio "piano organico e di ampio respiro per ripopolare le città siciliane decadute" 51 .

\section{RIFONDAZIONI/SINECISMI ALLE ORIGINI DELLA PROVINCIA: IL RUOLO DI ROMA}

Ho sin qui cercato di costruire un quadro generale di riferimento che possa aiutare a valutare, in mancanza di dati più specifici, i casi di Tindari, Solunto e Finziade: l'ipotesi che mi sembra più plausibile e che vorrei sottoporre all'opinione degli studiosi, è che si sia trattato di rifondazioni o, se si vuole, di sinecismi pilotati da Roma e non escluderei che siffatti interventi abbiano rappresentato anche l'occasione per stanziamenti di veterani. A tal proposito, a parte il caso ben noto della concessione di Morgantina agli Hispani nel 211 a.C. ${ }^{52}$, vale la pena di ricordare che proprio per Finziade Francesco La Torre ha suggerito la possibilità di riconoscere indizi della presenza di veterani di Marcello tra gli abitanti della "nuova" città: si tratta del rinvenimento di un tesoretto occultato dentro la muratura di una delle case al momento della sua costruzione, che secondo lo studioso si spiegherebbe nel modo più plausibile come lo stipendium che un veterano avrebbe murato per tesaurizzarlo, una volta stabilitosi nella nuova sede ${ }^{53}$.

L'attribuzione a Roma di un ruolo più incisivo in questi interventi non significa, naturalmente, voler omologare la complessità di moventi e implicazioni che dobbiamo immaginare dietro ognuno di essi, né, tanto meno, ammettere l'esistenza di una visione programmatica da parte dei vincitori; sull'effettiva esistenza di un piano organico da parte di Roma è preferibile, a mio parere, sospendere il giudizio.

(47) Crawford 1990, p. 93; Prag 2012, p. 59.

(48) Diod. 4.83.47; Cic. Verr. 5.124.

(49) Cfr. in proposito Prag 2007a, p. 82-83; Prag 2012, p. 62-63. Per una discussione sulle possibili datazioni si veda inoltre Prag 2011, p. 195-197, a favore di una data di poco successiva al 241 a.C.

(50) Da ultimo Prag 2014, p. 197, con ulteriori riferimenti.

(51) Gallo 1982, p. 932-933.

(52) Liv. 26.21.17.

(53) La Torre 2013, p. 425-426, 469-471. Sul tesoretto cfr. La Torre, Mollo 2013, p. 479-496 (Appendice di M. Caccamo Caltabiano, K. Longo, G. Salamone, A. De Miro). 
Ciononostante, non si può fare a meno di chiedersi se i tre centri esaminati siano gli unici da iscrivere in tale dossier. Esistono, come si è detto all'inizio, diversi altri esempi di città siciliane organizzate secondo una pianificazione regolare, per la cui redazione sono state proposte, caso per caso, datazioni successive alla metà del III sec. a.C.: basti pensare ai centri di Alesa, le cui somiglianze con l'impianto di Tindari e soprattutto di Solunto sono state più volte evidenziate, o di Eraclea Minoa, per la quale colpiscono invece le affinità con Finziade, ma l'elenco potrebbe allungarsi a comprendere almeno Panormos, Cefalù e Lilibeo ${ }^{54}$. Si è già osservato che tali proposte di datazione - per quanto, a mio parere, assai plausibili in molti casi - almeno per il momento non sono sostenute da dati stratigrafici dirimenti, e quasi ovunque non è possibile stabilire se l'impianto urbano regolare coincida con la fondazione o se, e in che misura, abbia modificato un insediamento già esistente.

Tuttavia, è persino banale evidenziare come i centri in questione ricadano tutti all'interno della vetus provincia e siano tutti centri costieri con una posizione assai rilevante sotto il profilo strategico o come punti di partenza per le rotte verso il Tirreno o verso l'Africa, per cui dal punto di vista di Roma non stupirebbe un interesse verso il rafforzamento di questi centri sin dalle prime fasi dopo la conquista dell'eparchia punica.

Più in generale, sin dalle prime fasi dopo la conquista, Roma dovette avere ben presente l'importanza dei centri urbani per la riorganizzazione del territorio isolano; almeno sotto questo profilo, il processo di provincializzazione della Sicilia sembra seguire modalità diverse da quelle che nello stesso arco di tempo caratterizzano la costituzione della provincia Sardinia et Corsica ${ }^{55}$. Rispetto alla Sicilia, per entrambe le isole lo stato delle conoscenze archeologiche sulle primissime fasi dopo la conquista è ancora più lacunoso; almeno fino a questo momento, però, non risultano casi di riorganizzazione degli insediamenti urbani prima della tarda età repubblicana, tali da configurare dinamiche simili a quelle qui proposte per la Sicilia ${ }^{56}$.

E tuttavia, perché si possa procedere ad un più fondato raffronto tra le due prime province, anche per la Sicilia è necessario disporre di dati archeologici più abbondanti, in grado di restituire alle forme urbane dei centri isolani tutto il loro spessore storico, andando oltre l'impostazione descrittiva e formale della quale gran parte della ricerca su questi temi è rimasta troppo a lungo prigioniera.

Lorenzo CAMPagna

Dipartimento di Civiltà Antiche e Moderne - Università di Messina

(54) Cfr. la bibliografia citata supra, nota 9. In particolare, per l'impianto urbano di Alesa, cfr. da ultimo Tigano 2016, che propende (part. p. 138) per una datazione tra la fine del IV e i primi decenni del III sec. a.C., probabilmente in seguito alla battaglia del Longano e al passaggio della città sotto il controllo di Ierone II di Siracusa. Tuttavia, almeno per la costruzione della mura - delle quali opportunamente la studiosa sottolinea la stretta relazione progettuale e cronologica con la pianificazione urbana - non sembra possibile escludere del tutto una datazione di poco successiva, in base ai materiali rinvenuti da Gianfilippo Carettoni in contesti utili per la datazione (cfr. CARETTONi 1959).

Non includerei invece nell'elenco Morgantina, il cui impianto regolare secondo gli scavatori risalirebbe (come per Agrigento: vedi supra, nota 4) ad epoca precedente: cfr. BeLl 2006 (metà-terzo quarto del V sec. a.C.). Andrebbero piuttosto considerati altri casi ancora poco noti sotto il profilo sia topografico che cronologico, come Lipari, Akrai o Adrano.

(55) Sulle prime fasi delle due province cfr., anche per ulteriori riferimenti, PRAG 2012.

(56) Si vedano al riguardo i contributi raccolti in De Vincenzo, Blasetti Fantauzzi 2016; in particolare, De VinCENZO 2016a. 


\section{Bibliografia}

Ampolo, C. (ed.), 2001, Da un'antica città di Sicilia. I decreti di Entella e Nakone. Catalogo della mostra, Pisa.

Anastasi, C., 1999, «Studi vecchi e nuovi sulle dinamiche migratorie nella Sicilia romana di eta repubblicana», in M. Barra Bagnasco, E. De Miro, A. Pinzone (eds.), Origine e incontri di culture nell'antichità. Magna Grecia e Sicilia. Stato degli studi e prospettive di ricerca (Pelorias, 4), Soveria Mannelli, p. 403-414.

BEJOR, G., 1983, «Aspetti della romanizzazione della Sicilia», in Modes de contacts et processus de transformation dans les sociétés anciennes. Actes du colloque de Cortone (24-30 mai 1981) (CÉFR 67), Rome, p. 344-374.

Bell III, M., 2006, «Rapporti urbanistici fra Camarina e Morgantina», in P.Pelagatti, G. Di Stefano, L. De Lachenal (eds.), Camarina 2600 anni dopo: nuovi studi sulla città e sul territorio. Atti del convegno internazionale (Ragusa, 7 dicembre 2002/7-9 aprile 2003), Roma, p. 253-258.

Belvedere, O., 1987, «Himera, Naxos e Camarina, tre casi di urbanistica coloniale», Xenia 14, p. 5-20.

Belvedere, O., 1998, «Introduzione», in Storia della Sicilia, I. La Sicilia antica, 1 (seconda edizione), Roma, p. XI-XXI.

Belvedere, O., Termine, E., 2005, «L'urbanizzazione della costa nord-orientale della Sicilia e la struttura urbana di Tindari», in S.T.A.M. Mols, E. Moormann (eds.), Omni pede stare. Saggi architettonici e circumvesuviani in memoriam Jos de Waele (Studi della Soprintendenza Archeologica di Pompei, 9), Napoli, p. 85-91.

Bernabò-Brea, L., Cavalier, M., 1965, «Tindari. Area urbana. L'insula IV e le strade che la circondano», BdA 50, p. 205-209.

Brienza, E., CAliò, L. M., 2018, «Urbanistica e scenografia del quartiere centrale di Agrigento tra età classica ed ellenistica», in V. Caminneci, M. C. Parello, M.S. Rizzo, C. Soraci (eds.), Agrigento ellenisticoromana. Coscienza identitaria e margini di autonomia (Atti della Giornata di Studi, Agrigento, 30 giugno 2016), Bari, p. 43-62.

Brienza, E., Caliò, L.M., Furcas, G.L. et al., 2016, «Per una nuova definizione della griglia urbana della antica Akragas. Contributo preliminare ad una nuova immagine della città», $\mathrm{ArchCl}$ 67, p. 57-109.

Brienza, E., Caliò, L. M., Lippolis, E., 2011, «Castiglione di Paludi: nuove ricerche nel sito della città antica», in G. De Sensi Sestito, S. Mancuso (eds.), Enotri e Bretti in Magna Grecia, Soveria Mannelli, p. 258-289.

CAmpagna, L., 2003, «La Sicilia di età repubblicana nella storiografia degli ultimi cinquant'anni», Ostraka XII, 1, p. 7-31.

CAmpagna, L., 2006, "L'architettura di età ellenistica in Sicilia: per una rilettura del quadro generale», in M. Torelli, M. Osanna (eds.), Sicilia ellenistica, consuetudo italica. Alle origini dell'architettura ellenistica d'Occidente (Atti delle giornate di studio, Spoleto, 5-7 novembre 2004), Roma, p. 15-34.

CAMPAgNA, L., 2007, «Architettura pubblica ed evergetismo nella Sicilia di età repubblicana», in C. MicCichè, S. Modeo, L. Santagati (eds.), La Sicilia romana tra Repubblica e Alto Impero (Atti del Convegno di Studi, Caltanisetta, 20-21 maggio 2006), Caltanissetta, p. 110-134.

Campagna, L., 2011, «Exploring social and cultural changes in Provincia Sicilia. Reflections on the study of urban landscapes», in F. Colivicchi (ed.), Local cultures of South Italy and Sicily in the Late Republican Period. Between Hellenism and Rome (JRA Supplementary series, 83), Portsmouth, p. 161-183.

Carettoni, G., «Tusa. Scavi di Halaesa (prima relazione)», NotSc, p. 293-349.

Caruso, E., 2013, «Lilibeo (Marsala), l'insula I di Capo Boeo. Eccezione urbanistica e monumentale domus urbana», SicArch 101, p. 153-164.

Caruso, E., 2014, «Lilibeo. Un esempio dell'urbanistica punica in Sicilia», in A. M. Arruda (ed.), Fenícios e Púnicos, por terra e mar. 2. Actas do VI Congresso Internacional de Estudos Fenício Púnicos (Lisboa 2005), Lisboa, p. 946-959.

Castagnoli, F., 1963, «Recenti ricerche sull'urbanistica ippodamea», ArchCl 15, p. 180-197.

Castagnoli, F., 1971, Orthogonal Town Planning in Antiquity, Cambridge MA - London.

Cavalier, M., «La stazione preistorica di Tindari», BPI XXI, 79, p. 61-93. 
Corsaro, M., 2003, «Prefetti romani in città provinciali: il caso di Entella», in A.M.CordA (ed.), Cultus splendore. Studi in onore di Giovanna Sotgiu, Senorbì, p. 373-396.

Corsaro, M., 2016, «La "questione sociale” nella Sicilia di Agatocle», in E. De Miro (ed.), Studi in memoria di Giacomo Manganaro, SicAnt XIII, p. 51-58.

Crawford, M.H., 1990, «Origini e sviluppi del sistema provinciale romano», in Storia di Roma, 2. L'impero mediterraneo, 1. La repubblica imperiale, Torino, p. 91-121.

De Vincenzo, S., 2013a, «Bemerkungen zu Urbanistik und Kultaspekten der Stadt Solunt in punischer und römischer Zeit», MedAnt 16, p. 767-794.

De Vincenzo, S., 2013b, Tra Cartagine e Roma. I centri urbani dell'eparchia punica di Sicilia tra VI e I sec. a.C. (Topoi 8), Berlin.

De Vincenzo, S., 2014, «Zwischen Griechen und Karthagern. Beitrag zur punischen Identität Selinunts im Kontext der Expansionspolitik Karthagos auf Sizilien», MedAnt 17, p. 235-267.

DE Vincenzo, S., 2016a, « Aspetti del processo di romanizzazione dei centri urbani della Sardegna in età tardorepubblicana», in De Vincenzo, Blasetti Fantauzzi 2016, p. 123-141.

De Vincenzo, S., 2016b, «Western Sicily from Romanization to the Punic age. Methodological problems and suggestions on the basis of some case studies», in Karthago Dialoge. Karthago und der punische Mittelmeerraum. Kulturkontakte und Kulturtransfers im 1. Jahrtausend vor Christus, Tübingen, p. 207226.

De Vincenzo, S., Blasetti Fantauzzi, C. (eds.), 2016, Il processo di romanizzazione della provincia Sardinia et Corsica. Atti del Convegno Internazionale di Studi, Cuglieri (OR) 26-28 marzo 2015 (Analysis Archaeologica. Monograph Series, 1), Roma.

Di Vita, A., 1996, "Urbanistica della Sicilia greca», in I Greci in Occidente (Catalogo della mostra, Venezia, Palazzo Grassi, marzo - dicembre 1996), Milano, p. 263-310.

Di Vita, A., 2002, «L'urbanistica nella Sicilia del IV secolo a.C.», in N. Bonacasa, L. Braccesi, E. De Miro (eds.), La Sicilia dei due Dionisî. Atti della settimana di studio (Agrigento 24 - 28 febbraio 1999), Roma, p. 139-146.

GABBA, E., 1959, «Sui senati delle città siciliane nell'età di Verre», Athenaeum 37, p. 304-320.

Gallo, L., 1982, «'Polyanthropia', 'eremia', e mescolanza etnica in Sicilia: il caso di Entella,», ASNP s. III, 12, p. 917-944.

Goldsberry, M.A., 1973, Sicily and its cities in Hellenistic and Roman times (diss., University of North Carolina), Chapel Hill.

Gruet, B., 2008, «Retour sur Hippodamos de Milet. À propos d'un mythe moderne», Histoire Urbaine 21, 1, p. 87-110.

ItAliA, A., LimA, D., 1987, «Solunto. Struttura urbana e tipologia residenziale», SicA XX, 65, p. 57-72.

LA Torre, G. F., 2004, «Il processo di romanizzazione della Sicilia. Il caso di Tindari», SicAnt 1, p. 111-146.

LA Torre, G.F., 2009, «Dal Phrourion alla Polis: città d'altura nella Sicilia ellenistica», in M. CongIU, S. Modeo, C. Miccichè (eds.), Eis Akra: insediamenti d'altura in Sicilia dalla Preistoria al III sec. a.C. (Atti del V convegno di studi, Caltanissetta 10-11 maggio 2008), Caltanissetta-Roma, p. 191-200.

LA Torre, G.F., 2013, «Dallo scavo alla ricostruzione storica», in LA Torre, Mollo 2013, p. 419-478.

La Torre, G. F., Mollo, F., (eds.), 2013, Finziade I. Scavi sul Monte Sant'Angelo di Licata (2003-2005), Roma. Lombardo, M., 1982, «Il sinecismo di Entella», ASNP s. III, 12, p. 849-886.

Lombardo, M., 2018, «Entella tra i Cartaginesi e i Romani, ovvero da chi erano stati espulsi gli Entellini?», in F. Camia, L. Del Monaco, M. Nocita (eds.), Munus Laetitiae. Studi miscellanei offerti a Maria Letizia Lazzarini, Roma, II, p. 485-498.

Manganaro, G., 1964, "Città di Sicilia e santuari panellenici nel III e II sec. a.C.», Historia 13, p. 416-439.

Manganaro, G., 1980, «La provincia romana», in E. Gabba, G. Vallet (eds.), La Sicilia antica II 2. La Sicilia romana, Napoli, p. 413-461. 
Marasco, G., 2007, «Les deux premières guerres puniques et la population de la Sicile», in J.-N. Corvisier (ed.), Guerre et démographie dans le Monde Antique (Colloque international d'Arras, 14-15 décembre 2001), Arras, p. 171-185.

Mattioli, M., 1995, «Roma e la Sicilia nel III sec a.C. Morgantina ed Entella», Acme 48, p. 5-21.

Medeksza, S., 1990, «Problèmes d'urbanisme et d'architecture de l'habitat hellénistique à Solunte», EtTrav 14, p. 91-130.

Merguet, H., 1877-1884, Lexikon zu den Reden des Cicero, Jena.

Mertens, D., 2006, Città e monumenti dei Greci d'Occidente. Dalla colonizzazione alla crisi di fine V secolo a.C., Roma.

Mezzolani, A., 1994, «Urbanistica regolare nel mondo punico. Note introduttive», in L. QUiLICI, S. QUILICI GigLi (edd.), Opere di assetto territoriale ed urbano (Atlante tematico di topografia antica, 3), Roma, p. 147-158.

Mistretta, A., 2016, «Il progetto Lilibeo. Genesi ed evoluzione di un centro punico-ellenistico nella Sicilia nord-occidentale», AntK 59, p. 123-131.

Moggi, M., 1975, «Synoikizein in Tucidide», ASNP 5,3, p. 915-924.

Pinzone, A., 1995, «A proposito di romanizzazione della Sicilia nell'età delle guerre puniche», in La Sicilia tra l'Egitto e Roma. La monetazione siracusana dell'età di Ierone II (Atti del seminario di studi, Messina 2 - 4 dicembre 1993), Messina, p. 475-493.

Pinzone, A., 1999, «Civitates sine foedere immunes ac liberae: a proposito di Cic. II Verr. III 6,13», MedAnt 2, p. 463-495.

Pinzone, A., 2000, "La romanizzazione della Sicilia occidentale in età repubblicana», in Atti delle Terze giornate internazionali di studi sull'area elima (Ghibellina - Erice - Contessa Entellina, 23-26 ottobre 1997), Pisa, p. 849-878.

Pinzone, A., 2004, «I socii navales siciliani», in M.Caccamo Caltabiano, L. Campagna, A. Pinzone (eds.), Nuove prospettive della ricerca sulla Sicilia del III sec. a.C.: archeologia, numismatica, storia (Atti dell'incontro di studio, Messina, 4-5 luglio 2002) (Pelorias, 11), Soveria Mannelli, p. 11-34.

Portale, E. C., 2001-2002, «Per una rilettura delle arti figurative nella Provincia Sicilia: pittura e mosaico tra continuità e discontinuità», SEIA n.s. 6-7, p. 43-90.

Portale, E. C., 2006, «Problemi dell'archeologia della Sicilia ellenistico-romana: il caso di Solunto», $\mathrm{ArchCl}$ 57, p. 49-114.

Portale, E. C., 2007, «A proposito di «romanizzazione» della Sicilia. Riflessioni sulla cultura figurativa», in C. Micciché et alii (eds.), La Sicilia romana tra Repubblica e Alto Impero (Atti del Convegno di Studi, Caltanissetta 20-21 maggio 2006), Caltanissetta, p. 150-169.

Portale, E. C., 2015, «Un confronto: la Sicilia nel III secolo», in La Magna Grecia da Pirro ad Annibale. Atti del LII Convegno di Studi sulla Magna Grecia, Taranto, p. 697-727.

Portale, E. C., 2017, «Siracusa e la Sicilia nel III secolo a.C.: problemi conoscitivi e proposte di lettura dei fenomeni urbanistici e architettonici» in L. M. CALIò, J.Des Courtils (eds.), L'architettura greca in Occidente nel III secolo a.C. Atti del Convegno di Studi, Pompei-Napoli, 20-22 maggio 2015, Roma, p. 133-177.

Prag, J.R.W., 2003, «Nouveaux regards sur les élites locales de la Sicile républicaine», Histoires et sociétés rurales 19, p. 121-132.

Prag, J.R.W., 2007a, «Auxilia and gymnasia: a Sicilian model of Roman Republican Imperialism», JRS 97, p. $68-100$.

Prag, J. R. W., 2007b, «Roman magistrates in Sicily, 227-49 B.C.», in La Sicile de Cicéron. Lectures des Verrines. Actes du Colloque de Paris, 19-20 mai 2006, Besançon, p. 287-310.

Prag, J.R.W., 2009, «Republican Sicily at the start of the 21st Century: the rise of the optimists», Pallas 79, p. 131-144. 
PraG, J.R.W., 2011, «Provincia Sicilia: between Roman and local in the third century BC», in E. GARCÍA Riaza (ed.), De Fronteras a provincias. Interacción e integración en Occidente (ss. III-I aC), Palma de Mallorca, p. 83-96.

Prag, J.R.W., 2012, «Sicily and Sardinia-Corsica: the first provinces», in D. Hoyos (ed.), A Companion to Roman Imperialism, Leiden, p. 53-65.

Prag, J.R.W., 2014, «Cities and civic life in late Hellenistic Roman Sicily (with an appendix on Cicero, In Verrem 3.12-13 and the status of cities in Sicily after 210 BC)», CahGlotz 25, p. 165-208.

RIzzo, F. P., 1968-1969, «Problemi costituzionali sicelioti», Kokalos XIV-XV, p. 365-393.

Rizzo, F.P., 1976-1977, "Malta e la Sicilia in età romana: aspetti di storia politica e costituzionale», Kokalos XXII-XXIII, p. 173-214.

Rocco, G., 2015, «L'architettura in Sicilia e in Magna Grecia tra ellenismo e romanizzazione», in La Magna Grecia da Pirro ad Annibale. Atti del LII Convegno di Studi sulla Magna Grecia, Taranto, p. 777-806.

Russenberger, C., 2016, «Punier am Berg. Archäologische Szenarien punischer Präsenz im Binnenland des frühhellenistischen Westsizilien am Beispiel des Monte Iato», in F.Schön, H.TöPfer (eds.), Karthago Dialoge. Karthago und der punische Mittelmeerraum. Kulturkontakte und Kulturtransfers im 1. Jahrtausend vor Christus, Tübingen, p. 227-251.

Russenberger, C., 2017, «Modalités archéologiques de la présence punique à l'intérieur de la Sicile occidentale au début de la période hellénistique: l'exemple de Monte Iato», in H. Dridi, D. Wieland-Leibundgut, J. Kraese (eds.), Phéniciens et Puniques en Méditerranée. L'apport de la recherche suisse. Phönizier und Punier im Mittelmeerraum. Ein Beitrag der Schweizer Forschung, Rome, p. 79-99.

Schmiedt, G., 1963, «Contributo della fotografia aerea alla ricostruzione della topografia antica di Lilibeo», Kokalos IX, p. 49-72.

Serrati, J., 2000, «Garrison and Grain: Sicily between the Punic Wars», in C. J. Smith, J. Serrati (eds.), Sicily from Aeneas to Augustus, Edinburgh, p. 115-133.

Sommella, P., 1987-1988, «Ippodamo di Mileto e l'urbanistica delle città greche in Sicilia», CronA 26-27, p. $79-88$.

SpataforA, F., 2009, «Dagli emporia fenici alle città puniche. Elementi di continuità e discontinuità nell'organizzazione urbanistica di Palermo e Solunto», in Phönizisches und punisches Städtewesen. Akten der internationalen Tagung in Rom vom 21. bis 23. Februar 2007, Mainz, p. 219-239.

SpIgo, U., 2005, «L'Insula IV: le case B e C, le terme romane, i mosaici», in U. SpIGo (ed.), Tindari. L'area archeologica e l'Antiquarium, p. 42-50.

SpIgo, U., 2006, «Tindari. Considerazioni sull'impianto urbano e notizie preliminari sulle recenti campagne di scavo nel settore occidentale», in M. Torelli, M. Osanna (eds.), Sicilia ellenistica, consuetudo italica. Alle origini dell'architettura ellenistica d'Occidente (Atti delle giornate di studio, Spoleto, 5-7 novembre 2004), Roma, p. 97-105.

SPIGo, U., 2008, «Le campagne di scavo 1993-2004. Contributi conoscitivi al quadro storico e culturale di Tyndaris e della Colonia Augusta Tyndaritanorum», in R. Leone, U. SPIgo (eds.), Tyndaris 1. Ricerche nel settore occidentale: campagne di scavo 1993-2004, Palermo, p. 101-111.

Tigano, G., 2016, «Alesa Arconidea. Appunti sull'impianto urbano alla luce della recenti ricerche», in E. Lattanzi, R. Spadea (eds.), Se cerchi la tua strada verso Itaca... Omaggio a Lina di Stefano, Roma, p. 129-142.

Toscano Raffa, A., Venuti, M., Papale, M., Miano, M., 2015, «Nuovi dati dall'abitato ellenistico-romano di Finziade sul Monte Sant'Angelo di Licata (AG). Gli scavi dei settori H (2012-2013), F (2014) e J (2014)», QuadMess n. s. V, p. 63-82.

TusA, V., 1999, «Testimonianze fenicio-puniche e urbanistica ippodamea a Solunto», in Koıv $\alpha$. Miscellanea di studi archeologici in onore di Piero Orlandini, Milano, p. 369-375. 
VAssallo, S., 2011, «Trasformazioni negli insediamenti della Sicilia centro-settentrionale tra la fine del V e il III secolo a.C. con nota preliminare sul teatro di prima età ellenistica di Montagna dei Cavalli», in Krise und Wandel. Süditalien im 4. und 3. Jahrhundert v.Chr. Internationaler Kongress anlässlich des 65. Geburtstages von Dieter Mertens (Rom 26. bis 28. Juni 2006), Wiesbaden, p. 55-77.

WiLson, R. J. A., 1985, "Changes in the Pattern of Urban Settlement in Roman, Byzantine and Arab Sicily», in C. Malone, S. Stoddart (edd.), The Human Landscape. Papers in Italian Archaeology IV.1 (BAR Int. Ser. 243), Oxford, p. 314-344.

Wilson, R. J. A., 2013a, «Hellenistic Sicily, c.270-100 BC», in J. R. W. Prag, J. C. Quinn (eds.), The Hellenistic West. Rethinking the Ancient Mediterranean, Cambridge, p. 79-119.

Wilson, R. J. A., 2013b, «Sicily c.300 BC-133 BC, in C. Smith (ed.), The Cambridge Ancient History. Plates to Volumes VII Part 2 and VIII. The Rise of Rome to 133 BC, New York, p. 156-196.

ZамвоN, E., 2008, Tradition and Innovation. Sicily between Hellenism and Rome, Stuttgart. 